\title{
Erratum
}

\section{Spontaneous Breaking of Anti-de Sitter Supersymmetry}

S. Bellucci

Elementary Particles Group, Department of Physics and Astronomy, University of Maryland, College Park, MD 20742, USA

Z. Phys. C.- Particles and Fields 33, 551-560 (1987)

The equations at the end of page 553 and at the beginning of page 554 should read as follows:

$$
\begin{aligned}
S_{D S}= & m G_{0}+i \not h \frac{\partial G_{0}}{\partial \mu}-i a^{2} \not h \frac{\partial}{\partial m^{2}} \frac{\partial}{\partial \mu} G_{0} \\
& -i \not h a^{2} m^{2} \frac{\partial^{2}}{\left(\partial m^{2}\right)} \frac{\partial}{\partial \mu} G_{0}-m a^{2} \frac{\partial G_{0}}{\partial m^{2}} \\
& -m^{3} a^{2} \frac{\partial^{2} G_{0}}{\left(\partial m^{2}\right)^{2}}+\mathcal{O}\left(a^{3}\right), \\
\mathscr{G}_{D S}= & G_{0}-a^{2} \frac{\partial G_{0}}{\partial m^{2}}-a^{2} m^{2} \frac{\partial^{2} G_{0}}{\left(\partial m^{2}\right)^{2}}+\mathcal{O}\left(a^{3}\right) .
\end{aligned}
$$

